appeared in ApJL v636 (Jan. 10, 2006)

\title{
The Effects of Metallicity and Grain Size on Gravitational Instabilities in Protoplanetary Disks
}

\author{
Kai Cai, Richard H. Durisen, Scott Michael, Aaron C. Boley \\ Astronomy Department, Indiana University, Bloomington, IN 47405 \\ kai@astro.indiana.edu \\ Annie C. Mejía \\ Department of Astronomy, University of Washington, Box 351580, Seattle, WA 98195-1580 \\ acmejia@astro. washington. edu \\ Megan K. Pickett \\ Department of Chemistry and Physics, Purdue University Calumet, 2200 169th St., \\ Hammond, IN 46323 \\ pickett@astro.calumet.purdue.edu \\ and \\ Paola D'Alessio \\ Centro de Radioastronomia y de Astrofisica, Apartado Postal 72-3, 58089 Morelia, \\ Michoacan, Mexico \\ p.dalessio@astrosmo.unam.mx
}

\begin{abstract}
Observational studies show that the probability of finding gas giant planets around a star increases with the star's metallicity. Our latest simulations of disks undergoing gravitational instabilities (GI's) with realistic radiative cooling indicate that protoplanetary disks with lower metallicity generally cool faster and thus show stronger overall GI-activity. More importantly, the global cooling times in our simulations are too long for disk fragmentation to occur, and the
\end{abstract}


disks do not fragment into dense protoplanetary clumps. Our results suggest that direct gas giant planet formation via disk instabilities is unlikely to be the mechanism that produced most observed planets. Nevertheless, GI's may still play an important role in a hybrid scenario, compatible with the observed metallicity trend, where structure created by GI's accelerates planet formation by core accretion.

Subject headings: accretion, accretion disks — hydrodynamics — instabilities — planetary systems: formation — planetary systems: protoplanetary disks

\section{INTRODUCTION}

The past decade has seen the discovery of over 150 exoplanets (http://www.obspm.fr/planets). One statistical trend that has emerged from these data is that the probability of finding a gas giant planet around a star with current techniques increases with the host star's metallicity (Santos et al. 2001; Fischer \& Valenti 2005). As shown by Fischer \& Valenti (2005), the high metal content of planet host stars seems to be primordial. Therefore, this trend, if real (Sozzetti et al. 2005), indicates that short-period gas giant planets are more likely to occur in metal-rich than in metal-poor protoplanetary disks. The two contending theories for gas giant formation are core accretion plus gas capture (Pollack et al. 1996) and disk instability (Boss 2002; Mayer et al. 2004). Calculations show that the metallicity relation can be explained within the framework of core accretion (e.g., Ida \& Lin 2004; Kornet et al. 2005). For disk instability, Boss (2002) finds that, in his three-dimensional hydrodynamics disk simulations with radiative cooling, clump formation by disk instability occurs for all metallicities over the range 0.1 to $10 \mathrm{Z}_{\odot}$, due to rapid cooling by convection (Boss 2004), and he attributes the abundance of short period gas giants around high metallicity stars to migration (Boss 2005), a mechanism also invoked by Ida \& Lin (2004) to explain part of the metallicity correlation. By contrast, Mejía (2004), who uses a somewhat more sophisticated treatment of radiative boundary conditions, finds much longer cooling times and no fragmentation into dense clumps in her disk instability simulations. Here we report results of new disk calculations based on Mejía's methods in which the opacity is varied by using different metallicities and grain sizes. Even over a much narrower range of metallicities than considered by Boss (2002), we find that the strength of the GI's does vary noticeably and that disk fragmentation is not seen for any metallicity or grain size tested. 


\section{METHODS}

We conduct protoplanetary disk simulations using the Indiana University Hydrodynamics Group code (see Pickett et al. 1998, 2000, 2003; Mejía 2004; Mejía et al. 2005), which solves the equations of hydrodynamics in conservative form on a cylindrical grid $(r, \phi, z)$ to second order in space and time using an ideal gas equation of state. Self-gravity and shock mediation by artificial bulk viscosity are included. Reflection symmetry is assumed about the equatorial plane, and free outflow boundaries are used along the top, outer, and inner edges of the grid.

We adopt the treatment of radiative physics detailed in Mejía (2004) with few modifications. Let $\tau_{R}$ be the optical depth, defined by using the Rosseland mean opacity measured vertically down from above. Energy flow in cells with $\tau_{R}>2 / 3$ is calculated in all three directions using flux-limited diffusion (Bodenheimer et al. 1990). Cells with $\tau_{R}<2 / 3$, in the disk atmosphere and in the outer disk, cool radiatively using an optically thin LTE emissivity. Atmosphere heating by high-altitude shocks and upward moving photons from the photosphere are included. In this paper, we also assume that an external envelope heated by the star (Natta 1993; D'Alessio, Calvet \& Hartmann 1997) shines vertically down on the disk. This IR irradiation is characterized by a black body flux with a temperature $T_{i r r}$. The optically thick and thin regions are coupled, over one or two cells, by an Eddington-like grey atmosphere fit that defines the boundary flux for the diffusion approximation. The opacities and molecular weights for a solar composition are from D'Alessio et al. (2001), with a power-law grain size distribution of $n(a) \sim a^{-3.5}$ ranging from $0.005 \mu \mathrm{m}$ to a largest grain size $a_{\max }$ that can be varied. To model variations in metallicity $\mathrm{Z}$, the mean opacities are multiplied by a factor $f_{\kappa}=\mathrm{Z} / \mathrm{Z}_{\odot}$, as was done by Boss (2002). Tests of our radiative scheme for a vertically stratified gas layer with a constant gravity, a constant input flux at the base, and a grey opacity show relaxation to an Eddington-like solution with the correct flux from the photospheric layers.

\section{SIMULATIONS}

\subsection{Initial Model and the Set of Simulations}

The initial axisymmetric model for all the calculations is the same as that used by Mejía et al. (2005). The central star is $0.5 M_{\odot}$, and the nearly Keplerian disk of $0.07 M_{\odot}$ has a surface density $\Sigma(r) \propto r^{-0.5}$ from 2.3 AU to 40 AU. The initial grid has $(256,128$, 32 ) cells in $(r, \phi, z)$ above the midplane. When the disk expands at the onset of GI's, the

grid is extended radially and vertically. The initial minimum value of the Toomre stability 
parameter $Q$ is about 1.5, and so the disk is marginally unstable to GI's. The initial model is seeded with low-amplitude random density noise. We use $T_{i r r}=15 \mathrm{~K}$, which is lower than the $50 \mathrm{~K}$ assumed in Boss (2002) because our larger and less massive disk is mostly stabilized by $T_{i r r}=50 \mathrm{~K}$. In this paper, we present simulations with four metallicities $\mathrm{Z}=$ $1 / 4 \mathrm{Z}_{\odot}$ (one-quarter solar metallicity), $1 / 2 \mathrm{Z}_{\odot}, \mathrm{Z}_{\odot}$, and $2 \mathrm{Z}_{\odot}$. The $1 / 4 \mathrm{Z}_{\odot}$ simulation was started from the $1 / 2 \mathrm{Z}_{\odot}$ disk after 13.0 outer rotation periods (ORPs) of evolution, to save computing resources. Here 1 ORP (about $250 \mathrm{yrs}$ ) is the initial rotation period at 33 AU. The varied metallicity cases use a maximum grain size $a_{\max }=1 \mu \mathrm{m}$ in the dust opacities. An additional simulation with $a_{\max }=1 \mathrm{~mm}$ and $\mathrm{Z}=\mathrm{Z}_{\odot}$ is conducted to explore the effects of grain growth.

\subsection{Results}

The current calculations resemble those presented in Mejía (2004) and Mejía et al. (2005). The disks remain fairly axisymmetric until a burst phase of rapid growth in nonaxisymmetric structure. Subsequently, the disks gradually transition into a quasi-steady asymptotic phase, where heating and cooling are in rough balance, and average quantities change slowly (see also Lodato \& Rice 2005). Table 1 summarizes some of the results. In the table, Duration refers to the simulation length measured in ORPs, $t_{1}$ is the time in ORPs at which the burst phase begins, $t_{2}$ is the approximate time in ORPs when the simulation enters the asymptotic state, $\langle A\rangle$ is a time-averaged integrated Fourier amplitude for all nonaxisymmetric structure (see below), $t_{\text {cool }}$ is the final global cooling time obtained by dividing the final total internal energy of the disk by the final total luminosity, and Thin $\%$ is the percentage of disk volume that is optically thin during the asymptotic phase.

One noticeable effect is that the onset of the burst phase $\left(t_{1}\right)$ is delayed for higher metallicity and larger grain size (Table 1), as expected due to higher opacity and therefore slower cooling. Note that, over the bulk of our large cool disk, increasing $a_{\text {max }}$ increases the opacity. Although the time to reach the asymptotic phase is relatively insensitive to grain size and metallicity, the overall final $t_{c o o l}$ listed in Table 1 illustrates that the correlation between cooling time and opacity carries over to late times. During the asymptotic phase, in all cases, the Toomre $Q$ values remain roughly constant with time, with values ranging between 1.3 to 1.8 for $r=10$ to $40 \mathrm{AU}$, and the mass inflow rates peak near $15 \mathrm{AU}$ at $\sim 10^{-6} M_{\odot} / \mathrm{yr}$, with negligible difference between $1 / 2 \mathrm{Z}_{\odot}$ and $2 \mathrm{Z}_{\odot}$ to the accuracy that we can measure these inflows (Mejía et al. 2005). Although there are some regions of superadiabatic gradients, the rapid overall convective cooling reported by Boss $(2002,2004)$ does not occur. We do see

upward and downward motions, which we attribute to hydraulic jumps (Boley \& Durisen 
2006). Whether or not some of these motions are actually thermal convection, they do not result in rapid cooling for our disks.

In Figure 1, which shows midplane densities at 15 ORPs, the spiral structure appears stronger for $1 / 4 \mathrm{Z}_{\odot}$ than for $2 \mathrm{Z}_{\odot}$. In order to quantify differences in GI strength, we compute integrated Fourier amplitudes (Imamura et al. 2000)

$$
A_{m}=\frac{\int \rho_{m} r d r d z}{\int \rho_{0} r d r d z},
$$

where $\rho_{0}$ is the axisymmetric component of the density and $\rho_{m}$ is the amplitude of the $\cos (m \phi)$ component. Although variable, after $\sim 14$ ORPs, the $A_{m}$ 's for most $m$ 's are greater for $1 / 4 \mathrm{Z}_{\odot}$ than for higher Z's. To measure total nonaxisymmetry, we sum the $A_{m}$ 's and average this sum over 14.5 to 15.5 ORPs. As shown in Table 1, this global measure $\langle A\rangle$ is greatest for $1 / 4 \mathrm{Z}_{\odot}$ and generally decreases with increasing metallicity and grain size.

Figure 2 plots the cumulative energy loss due to cooling computed for only half the disk as a function of time. The upper curves show energy loss from the disk interior after compensating for energy input by residual irradiation and by the glowing disk upper atmosphere; the lower curves show net energy loss from optically thin regions after accounting for heating due to envelope irradiation. Due to our restricted vertical resolution and use of the Eddington atmosphere fit over one or two vertical cells, the "thick" curves effectively include most of the photospheric layers for most columns through the disk. The "thin" curves tally additional cooling from extended layers above the photospheric cells, usually with $\tau_{R}<<1$, plus the parts of the outer disk that are optically thin all the way to the midplane. The initial cooling rates for the optically thick regions plus photosphere clearly differ. In fact, the initial slopes of the "thick" curves give $t_{\text {cool }} \sim \mathrm{Z} / \mathrm{Z}_{\odot}$ ORPs. However, the initial disks are far from radiatively relaxed, and so there are transients. Remarkably, by the asymptotic phase, all the disk interior-plus-photosphere curves converge to similar energy loss rates. During these late times, the differences between the total cooling rates are dominated by the optically thin regions, which are larger for the lower metallicity cases, as indicated by the Thin\% entry in Table 1 . The overall asymptotic phase $t_{\text {cool }}$ 's in Table 1, based on summing the thick and thin loss rates, are longer for higher metallicity and larger grain size. Altogether, the evidence in Table 1 and Figures 1 and 2 shows that higher opacity leads to slower cooling and that slower cooling produces lower GI amplitudes. We remind the reader that we detect these differences over a much narrower range of metallicities $\left(1 / 4\right.$ to $\left.2 Z_{\odot}\right)$ than considered by Boss (2002) (0.1 to $\left.10 \mathrm{Z}_{\odot}\right)$.

As in Mejía (2004), except for brief transients during the burst phases of some runs, these disks do not form dense clumps, in apparent disagreement with Boss (2002). To investigate 
whether the disk evolution depends on spatial resolution in the asymptotic phase (Boss 2000, 2005; Pickett et al. 2003), both the $1 / 4 \mathrm{Z}_{\odot}$ and $2 \mathrm{Z}_{\odot}$ simulations are extended for another 2 ORP's with quadrupled azimuthal resolution (512 zones), and the disks do not fragment into dense clumps. This is consistent with the analytic arguments in Rafikov (2005) that an unstable disk and fast radiative cooling are incompatible constraints for realistic disks at $10 \mathrm{AU}$ (see Boss 2005 for a different perspective). Indeed, if $t_{\text {cool }}$ listed in Table 1 is a good measure of local cooling times in these disks, we do not expect fragmentation. Gammie (2001) shows that fragmentation occurs only if the local $t_{\text {cool }}$ is less than about half the local disk orbit period $P_{\text {rot }}$ (see also Rice et al. 2003; Mejía et al. 2005), except possibly near sharp opacity edges (Johnson \& Gammie 2003). We only find locallized cooling times shorter than $0.5 P_{\text {rot }}$ in the asymptotic phase of the $2 \mathrm{Z}_{\odot}$ case, and then only in the 30 to 40

AU region, which is optically thin. This occurs because, even though $t_{\text {cool }} \sim \mathrm{Z}$ in optically thick regions (higher optical depth), $t_{\text {cool }} \sim \mathrm{Z}^{-1}$ in thin ones (more emitters). As a result, this disk displays the steepest drop of local $t_{\text {cool }}$ with $r$. The short local $t_{\text {cool }}$ 's appear to be highly variable and transient. The continuation of this simulation for 2 ORPs at higher azimuthal resolution (512 zones) does not show evidence for fragmentation into clumps. It could prove important to push our simulations to higher $\mathrm{Z}$ in the future.

\section{DISCUSSION}

Our results show that GI strength decreases as metallicity increases and, contrary to Boss (2002), that global radiative cooling is too slow for fragmentation into dense clumps. In the asymptotic phase, cooling rates for the disk interior plus photospheric layers converge for all Z, but the total cooling, including the optically thin regions, is higher for lower Z. Thus, the optically thin upper atmosphere and outer disk play a role in the cooling rate of an evolved disk. In fact, the fractional volume of the optically thin regions becomes very large at late times (see Thin\% in Table 1). Note also that the optically thick region fractional volume, 1 - Thin \%, varies roughly as Z. The greater surface area of the disk photosphere at higher $\mathrm{Z}$ tends to compensate for the higher opacity and leads to convergence of the cooling rates for the parts of the disk contained within the photospheric layers. In this respect, we confirm Boss's conclusion that the outcome of the radiative evolution is somewhat insensitive to metallicity. However, the important difference is that we do not see fragmentation into dense clumps, presumably because our cooling rates are much lower than in Boss (2002). For the $1 \mathrm{~mm}$ case, the optically thin regions have a much smaller volume (Table 1) and contribute little to cooling. Outside the inner few AU, bigger grains make the disk more opaque to longer wavelengths, and $t_{\text {cool }}$ is thus considerably larger, even initially. 
Our results argue against direct formation of gas giants by disk instability in two ways - the global radiative cooling times seem too long for fragmentation to occur and GI's are stronger overall for lower metallicity. Nevertheless, it is still possible that GI's play an important role in gas giant planet formation. Durisen et al. (2005) suggest that dense gas rings produced by GI's will enhance the growth rate of solid cores by drawing solids toward their centers (Haghighipour \& Boss 2003) and thereby accelerating core accretion. Such rings are indeed produced in the inner disks of all our calculations regardless of metallicity or grain size, and they appear to be still growing when the calculations end. In the weaker GI environments of high metallicity, there is less self-gravitating turbulence to interfere with the radial drift of solids (Durisen et al. 2005). In this way, rings may provide a natural shelter and gathering place for growing embryos and cores.

The apparent disagreement between our results and those of Boss (2002, 2004) could be due to any number of differences in techniques and assumptions, such as artificial viscosity, opacities, equations of state, initial disk models and perturbations, grid shapes and resolution, and radiative boundary conditions, including the way that we handle irradiation. We are now collaborating with Boss in an effort to pinpoint which of these is the principal cause (K. Cai et al., in preparation). Preliminary results suggest that it is the radiative boundary conditions. We are therefore developing alternative techniques for disk radiative transfer that we hope are more reliable and accurate.

We thank A.P. Boss and an anonymous referee for useful comments. This work was supported in part by NASA Origins of Solar Systems grants Nos. NAG5-11964 and NNG05GN11G, by NASA Planetary Geology and Geophysics grant No. NAG5-10262, and by a Shared University Research grant from IBM, Inc. to Indiana University. 


\section{REFERENCES}

Bodenheimer, P., Yorke, H.W., Różyczka, M., \& Tohline, J.E., 1990, ApJ, 355, 651

Boley, A.C., \& Durisen, R.H., 2006, ApJ, in press (astro-ph 0510305)

Boss, A.P., 2000, ApJ, 536, L101

Boss, A.P., 2002, ApJ, 567, L149

Boss, A.P., 2004, ApJ, 610, 456

Boss, A.P., 2005, ApJ, 629, 535

D’Alessio, P., Calvet, N., \& Hartmann, L., 1997, ApJ, 474, 397

D'Alessio, P., Calvet, N., \& Hartmann, L., 2001, ApJ, 553, 321

Durisen, R.H., Cai, K., Mejía, A.C., \& Pickett, M.K., 2005, Icarus, 173, 417

Fischer, D.A., \& Valenti, J., ApJ, 622, 1102

Gammie, C.F., 2001, ApJ, 553, 174

Haghighipour, N., \& Boss, A.P., 2003, ApJ, 583, 996

Ida, S., \& Lin, D.N.C., ApJ, 616, 567

Imamura, J.N., Durisen, R.H., \& Pickett, B.K., 2000, ApJ, 528, 946

Johnson, B.M., \& Gammie, C.F., 2003, ApJ, 597, 131

Kornet, K., Bodenheimer, P., Różyczka, M., \& Stepinski, T.F., 2005, A\&A, 430, 1133

Lodato, G., \& Rice, W.K.M., MNRAS, 358, 1489

Mayer, L., Quinn, T., Wadsley, J., \& Stadel, J., 2004, ApJ, 609, 1045

Mejía, A.C., Ph.D. dissertation, Indiana University

Mejía, A.C., Durisen, R.H., Pickett, M.K., \& Cai, K., 2005, ApJ, 619, 1098

Natta, A., 1993, ApJ, 412, 761

Pickett, B.K., Cassen, P.M., Durisen, R.H., \& Link, R. 1998, ApJ, 504, 468

Pickett, B.K., Cassen, P.M., Durisen, R.H., \& Link, R. 2000, ApJ, 529, 1034 
Pickett, B.K., Mejía, A.C., Durisen, R.H., Cassen, P.M., Berry, D.K., \& Link, R.P. 2003, ApJ, 590, 1060

Pollack, J.B., Hubickyj, O., Bodenheimer, P. Lissauer, J.J., Podolak, M., \& Greenzwieg, Y., 1996, Icarus, 124, 62

Rafikov, R.R. 2005, ApJ, 621, L69

Rice, W.K.M., Armitage, P.J., Bate, M.R., \& Bonnell, I.A., 2003, MNRAS, 339, 1025

Santos. N. C., Israelian, G., \& Mayor, M., 2001, A\&A, 373, 1019

Sozzetti, A., Latham, D. W., Torres, G., Stefanik, R. P., Boss, A. P., Carney, B. W., \& Laird, J. B., in Proceedings of the Gaia Symposium "The Three-Dimensional Universe with Gaia" (ESA SP-576). Editors: C. Turon, K.S. O'Flaherty, M.A.C. Perryman., 309 
Table 1: Simulation Results

\begin{tabular}{|c|c|c|c|c|c|c|c|c|}
\hline \hline Case & $f_{\kappa}$ & $a_{\max }$ & Duration $^{\mathrm{a}}$ & $t_{1}^{\mathrm{a}}$ & $t_{2}{ }^{\mathrm{a}}$ & $\langle A\rangle$ & $t_{\text {cool }}{ }^{\mathrm{a}}$ & Thin\% \\
\hline $1 / 4 \mathrm{Z}_{\odot}$ & $1 / 4$ & $1 \mu \mathrm{m}$ & $3.8^{\mathrm{b}}$ & $\mathrm{N} / \mathrm{A}$ & $\mathrm{N} / \mathrm{A}$ & 1.29 & 2.1 & $99 \%$ \\
$1 / 2 \mathrm{Z}_{\odot}$ & $1 / 2$ & $1 \mu \mathrm{m}$ & 15.6 & 4.0 & 10 & 1.09 & 2.9 & $98 \%$ \\
$\mathrm{Z}_{\odot}$ & 1.0 & $1 \mu \mathrm{m}$ & 15.7 & 5.0 & 10 & 1.10 & 3.2 & $94 \%$ \\
$2 \mathrm{Z}_{\odot}$ & 2.0 & $1 \mu \mathrm{m}$ & 16.5 & 5.0 & 10 & 0.72 & 3.7 & $86 \%$ \\
$1 \mathrm{~mm}$ & 1.0 & $1 \mathrm{~mm}$ & 17.2 & 7.0 & 11 & 0.88 & 4.5 & $44 \%$ \\
\hline
\end{tabular}

${ }^{a}$ All times are given in units of ORPs.

${ }^{b}$ From 13.0-16.8 (ORPs). 


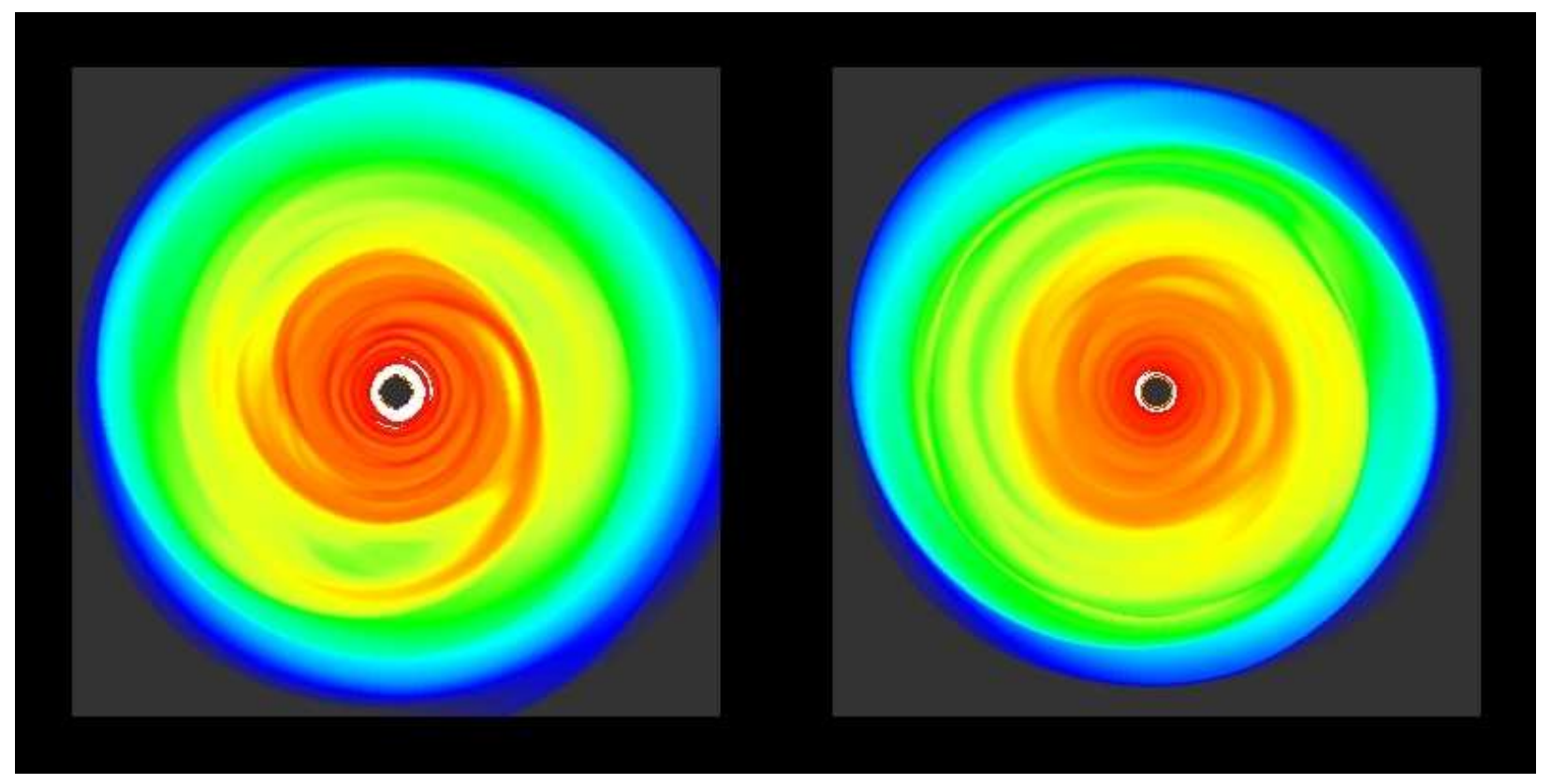

Fig. 1. - Midplane density maps at 15 ORPs for the $1 / 4 \mathrm{Z}_{\odot}$ (left panel) and $2 \mathrm{Z}_{\odot}$ (right panel) simulations. Each square is $113 \mathrm{AU}$ on a side. Densities are displayed on a logarithmic scale running from light grey to black (print version) or dark blue to dark red (online version), as densities range from about $4.8 \times 10^{-16}$ to $4.8 \times 10^{-11} \mathrm{~g} \mathrm{~cm}^{-3}$, respectively, except that both scales saturate to white at even higher densities. 


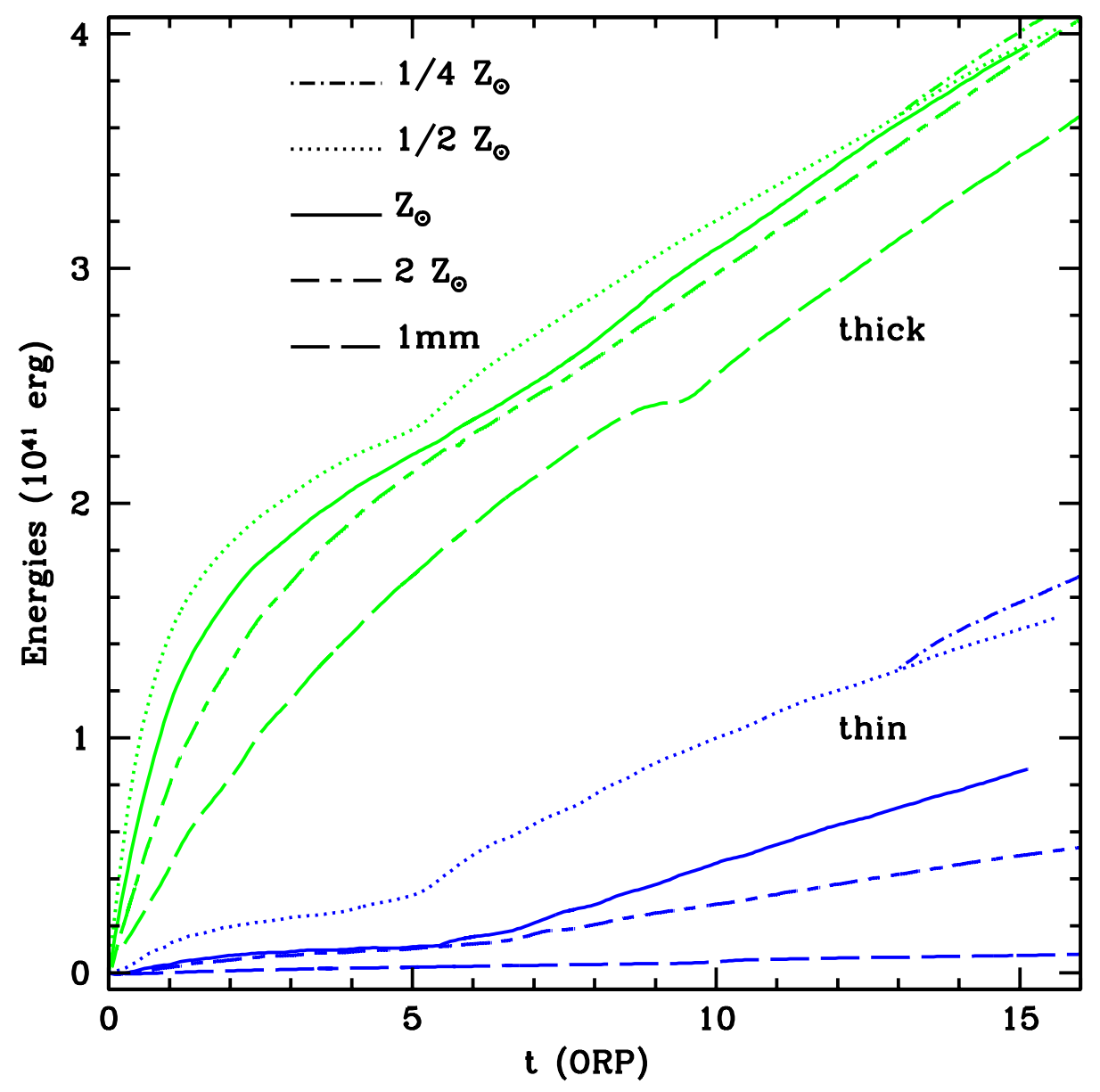

Fig. 2.- Cumulative total energy loss as a function of time due to radiative cooling in optically thick (upper set, labelled "thick") and optically thin regions (lower set, labelled "thin"). Both of these are net global cooling after heating by irradiation is subtracted. The curves labeled by a metallicity value all use $a_{\max }=1 \mu \mathrm{m}$. The curves labeled " $1 \mathrm{~mm}$ " are for a calculation with $a_{\max }=1 \mathrm{~mm}$ and solar metallicity. Note that the $1 / 4 \mathrm{Z}_{\odot}$ run starts from the $1 / 2 \mathrm{Z}_{\odot}$ simulation at about 13 ORPs. A color version of this figure appears on line. 